\title{
The Role of Physical Activity and Physical Therapy on Muscle Relaxation and Pain in Neck Disorders
}

\author{
Asuman SALTAN ${ }^{1}$, Yeşim BAKAR ${ }^{2}$, Eylem TÜTÜN YÜMİN ${ }^{3}$, \\ Meral SERTEL $^{4}$, Necati TATARLI ${ }^{5}$, Handan ANKARALI ${ }^{6}$
}

\begin{abstract}
Background: Musculoskeletal problems (neck, shoulder, back pain etc.) occur as a chronic pain or disability that reducesquality of life and economic productivity. To test the hypothesis that, comparison of the effectiveness of one-session application of two physiotherapy methods. The other aim is to investigation the relationship between physical activity and pain, muscle relaxation. Method: This study was designed randomized trial and double blind. Participants (with neck pain) divided into two groups (Classical Massage (CM), n=24 and Active Stretching, $n=21$ ) Pain were determined by the visual analog scale (VAS), a digital pressure algometer was used to assess pain threshold (PT) level and muscle relaxation response was measured by means of electromyography biofeedback (EMG_BF). Physical activity was determined by the physical activity assessment questionnaire (PAAQ). Results: Only the EMG values on the right SCM muscles of the CM group was found significantly higher than stretching group ( $\mathrm{p}=0.003)$. The relationship between EMG-BF, PT values and PAAQ was examined. Conclusions: Self-stretching is more effective than CM in providing muscle relaxation. Physical activities (especially steps,sitting at home working and school activities) particularly affect neck pain and SCM muscle.
\end{abstract}

Keywords: stretching, massage, electromyography feedback, pain threshold.

\section{Rezumat}

Introducere: Problemele musculo-scheletale (gât, umăr, durerea de spate etc.) se manifestă ca durere cronică sau dizabilitatecare reduce calitatea vieții și productivitatea economică. Scopul acestui studiu este de a verifica eficiența aplicării unei sesiuni unice a 2 metode fizioterapice; celălalt scop al acestui studiu este acela de a investiga relația dintre activitatea fizică și durere, relaxarea musculară. Metodă: Acest studiu a fost conceput randomizat și dublu-orb. Participanții (cu durere la nivelul gâtului) au fost împărțiți în 2 grupe (masaj clasic (CM), n=24 și stretching activ, n=21). Durerea a fost determinată cu scala vizuală analogică (VAS), un algometru cu presiune digitală a fost utilizat pentru a determina pragul la durere (PT), iar răspunsul privitor la relaxarea musculară a fost evaluat folosind biofeedback-ul electromiografic (EMG_BF). Activitatea fizică a fost determinată cu chestionarul de evaluare al activității fizice (PAAQ). Rezultate: Doar valorile EMG la mușchiul sternocleidomastoidian drept al grupului CM au fost semnificativ mai mari decât la grupul cu stretching $(\mathrm{p}=0.003)$. Relația dintre valorile EMG-BF, PT și PAAQ au fost examinate. Concluzii: Auto-stretching-ul este mai eficient decât masajul clasic în producerea relaxării musculare. Activitățile fizice (în special, urcatul scărilor, poziția șezând în activitățile casnice sau la școală) afectează în mod particular regiunea gâtului și mușchii sternocleidomastoidieni.

Cuvinte-cheie: stretching, masaj, feedback electromiografic, pragul durerii.

\footnotetext{
${ }^{1}$ Assistant Professor, PhD, PT, Yalova University Termal Vocational School,Yalova, TURKEY, e-mail: fzt_asuman@yahoo.com.tr

2 Associate Professor, PhD, PT, University of Abant İzzet Baysal, KD School of Physical Therapy and Rehabilitation, Bolu, Turkey.

${ }^{3}$ Assistant Professor, PhD, PT, University of Abant İzzet Baysal, KD School of Physical Therapy and Rehabilitation, Bolu, Turkey.

${ }^{4}$ Assistant Professor, PhD, PT, University Kırıkkale, Faculty of Health Sciences, Department of physiotherapy and rehabilitation, Kırıkkale, Turkey

5 Professor MD, University of Düzce, Faculty of Medicine,Department of Biyostatistic. Düzce, Turkey.

${ }^{6}$ MD, Lütfi Kırdar Education and Research Hospital, İstanbul, Turkey
} 


\section{Introduction}

Musculoskeletal disorders are an important health problem, common in both genders, affecting all age groups and resulting in loss of strength. Musculoskeletal problems (neck, shoulder and back pain etc.) occur as a chronic pain or disability that reduces quality of life and economic productivity [1,2]. Cervical musculoskeletal disorders are generally concomitant with back pain. The complaints generally start with pain, tension and induration and gradually turn into postural disruption, restriction of normal body movements and degeneration in the muscular and osseous structure. This situation reduces the quality of life and economic productivity of the individual. Therefore, musculoskeletal problems constitute an economic burden on society, both directly and indirectly $[3,4,5,6]$.

The impact of pain on daily living activies can be defined as a patien's disability level or decreased physical function. A decreased physical acivity level in daily life (disuse) has been presented as a perpetuating factor for chronicity in theoretical research models on pain. Although there is no exact results about physical activity in patients with chronic neck pain, there is controversial results on that physical activity must be increased or reduced in studies for chronic low back pain $[7,8]$.

Neck pain is a common complaint, with $0-18 \%$ point prevalence and $30-50 \%$ lifelong prevalence [9]. Various methods such as medicines, physical therapy, manual therapy, local or epidural injections and patient education are frequently used in the treatment of patients with neck pains [10]. Previous systematic reviews have not provided conclusive evidence on which of these methods is most effective $[11,12]$.

Massage and stretching exercises are two of the practical applications used in the rehabilitation of back pain [13]. Classical massage (CM), arterial blood flow, venous connection and blood enzyme concentrations have an impact on edemas, affect connective tissues and reduce muscle spasms, thus providing relaxation [14,15]. On the other hand, stretching is known to have an effect on the muscle fibril and provide relaxation and elasticity [16]. In addition, measurements taken directly after stretching applications show that it has neurological and hypoalgesic effects [17].
According to Good, AP et al [3], neck pain may cause absenteeism in working life as frequently as LBP. While neck pain has become such a serious health problem, little is known about the treatment of chronic neck pain. The researchers are inclined to treat their patients with evidence-based treatments such as massage, manipulative therapy and injection just because those treatments are easy to apply even if those treatments are ineffective and have less support in literature. ${ }^{3}$ In addition to the above, another systematic review suggests that massage is both a safe and widely used treatment for neck pain and any side effects are temporary and harmless. However, neither massage alone nor massage combined with other treatments (acupuncture, exercises, sham laser, transcutaneous electrical nerve stimulation, manual traction, mobilization, education and pain medication) showed a significant advantage over other comparison groups $[18,19]$. The European Guidelines do not recommend the use of any specific exercise programs such as stretching, strengthening, flexion or extension exercises for acute back pain. For subacute and chronic back pain and neck pain, there is limited evidence for the use of any specific exercise programs and in most guidelines no program is recommended [11].

Most studies on physical activity in pain patients have focused on people with chronic low back pain [20]. Less is known about the activity of patients with other types of chronic pain. Besides, not all aspects of daily activity were investigated together. Therefore, there is need a lot of study about relationship physical activity and pain [20].

The main purpose of this study was to investigate role of physical activity on neck pain and muscle relaxation. Another aims to investigate the effects of massage and exercise (active stretching), which are two methods easily used for neck and back pain on muscle relaxation and pain.

\section{Materials and Methods}

The study included 68 patients presenting to Köroglu State Hospital Neurosurgery Polyclinic with neck pain complaint, all of whom volunteered to take part in the research. As randomized trials, we divided two groups all participants. The CM group comprised 24 participants, while the active stretching group consisted of 21 participants. 
Diagnoses of the volunteers were carried out by a neurosurgeon.

Each participant completed an Informed Consent Form, which included an explanation of the purpose of the study.

The study inclusion criteria were: $25-45$ years of age, having neck pain for 3 to 6 months. The exclusion criteria were: disk hernia, stenosis, trauma history, cancer, osteoporosis, hypermobility, inflammatory rheumatologic diseases, severe psychological disorders; and being pregnant. After applying the inclusion and exclusion criteria, those included were referred to the physiotherapy department. Of the 68 patients, 23 patients did not meet the inclusion criteria (13 patients were older than 45 years, 7 patients had neck pain for more 6 months and 3 patients were not volunteers) (figure 1).

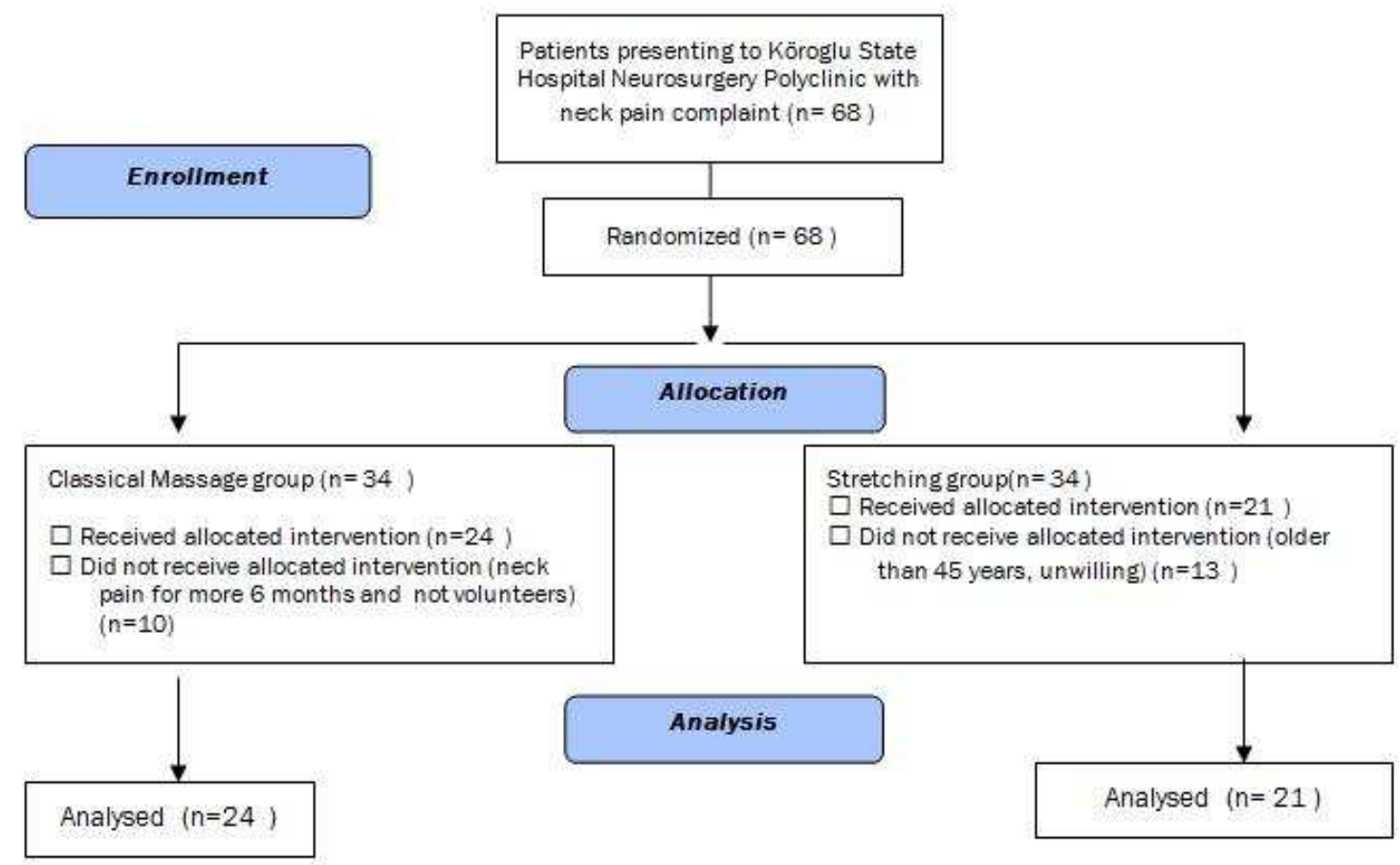

Figure 1. Flow diagram reflecting participants involvement in accordance with the CONSORT guidelines [30]

The study was designed as randomized and blind. The volunteers were randomly separated into two groups. Block randomization was by a computergenerated random number list prepared by an investigator with no clinical involvement in the trial. Patients were assessed immediately before and after each application by an independent (blinded) investigator. The applications were carried out for one session.

This study was approved by Bolu Clinic Research Ethics Board (2010/26).

An assessment form was prepared to collect the demographic data of the volunteers, such as age, height, weight and information such as job, marital status, and educational status.
A digital pressure algometer (JTECH MEDICAL, MonsterMarketplace.com®, (C) 2006-2011) was used to assess pain threshold (PT) levels. This device is a digital pain threshold meter that comprises a sensor connected to a hard edge with 1 $\mathrm{cm}$ diameter. The device measures $\mathrm{PT}$ as $\mathrm{lb} / \mathrm{cm} 2$. The instrument was applied to the patients' bare skin and the trigger motor point for Sternocleidomastoideus (SCM) and Trapezius (TR) muscles.

Measurement for the TR muscle was taken on the upper border of the TR muscle half-away between the midline and lateral border of the acromion. Measurements for the SCM muscle were taken on the SCM muscle between anterior, superior 
manubrium and superior medial third of clavicle and lateral aspect of mastoid process, anterior half of superior nuchal line.

These testing sites were chosen as they are known, through clinical experience, to be sensitive in patients with chronic non-specific neck pain. They were also used in all trials, as only intratester repeatability in reassessing pain sites qualitatively has been shown to be fair [21].

To measure PT value, the edge of the algometer was pressed on sensitive points until the maximum level that patients could bear. The patients were instructed to say "stop" at the point where the pressure became painful. The measurement was repeated three times, at 30 -second intervals, and the average of three measurements was taken; all values were expressed as $\mathrm{kg} / \mathrm{cm}^{2}$ unit [22].

The PT measurements were repeated in the same order at the same time by the same tester to evaluate the repeatability of the method. All measurements were performed by the same physiotherapist, who had several years' experience in testing. The algometer maintains the maximum applied pressure until tared. Thus, the measurements were performed blind, as the display was not in view of the tester and the peak output was read only after each measurement.

Muscle relaxation response was measured by means of electromyography (Myomed 932 EMG Biofeedback; Enraf Nanius, Rotterdam, Netherlans) [23]. For EMG (Electromyography Biofeedback) measurement from SCM, one of the active electrodes was placed under the Proc. Mastoideus, while the other was placed in the muscle's origin. For EMG measurement from TR muscle, the active electrodes were placed on the right and left TR muscle, along the lines connecting the spinous process of the seventh cervical vertebra (C7) to the right and left acromion, respectively. The passive electrode was placed on the upper extremity. Surface electrodes were used for the measurements. While carrying out measurements, all the volunteers were in a semi-horizontal position, supported by pillows in the front, and with the upper neck relaxed. During measurements, the volunteers were instructed to relax and not to move or talk.

The assessment form, PAAQ (Physical Activity Assessment Questionnaire) and VAS were used only at the beginning of the study, while PT and EMG-BF measurements were taken before and immediately after the application.

A visual analog scale (VAS) was used in subjective assessment of volunteers' pain. VAS is a simple and effective pain measurement scale with established validity and reliability. In order to determine body pain, volunteers were required to mark their pain levels on the $10 \mathrm{~cm}$ long scale, between "0" (no pain) and "10" (unbearable pain). The patients were instructed in how to use this scale [24].

Measure of physical activity: The PAAQ was originally developed by Karaca et al [25] to measure the physical activity level of individuals in the Turkish population. The PAAQ consists of six sub-scales of activities in which the individuals are expected to engage during a week. These sub-scales are related to activities involved in work, school, hobbies, home, transportation, climbing stairs and sports. For each subscale, participants were asked to report frequency and duration of the given activity. Total scores were calculated for each individual by using the syntax prepared by the test developer; the higher the scores, the more physically active they are. The measurement unit for the scale was MET/hour, in which MET stands for "metabolic equivalent" and is defined as the energy expenditure for sitting quietly. MET values can be converted to kcal [26].

In the classical massage group: Classic "Swedish massage" technique was applied to the upper back area for 20 minutes, focusing especially on SCM and TR muscles. The application was supported by frontward pillows and carried out in a frontward semi-horizontal position in which the upper back was relaxed [27].

In the stretching group: Participants were given a demonstration, by an examiner, on performing active neck flexion, neck lateral flexion, and neck rotation while seated near the edge of an armless chair with both feet firmly planted on the floor. ${ }^{28}$ Participants performed each of the following stretches, holding each movement for eight to 10 seconds; each exercise was repeated 10 times: $\mathrm{R}$ (right)/L (left) upper TR: hold on to the edge of the chair on the R/L side to keep the R/L shoulder from elevating. Side bend your head to the L/R. Gently pull your head over to the $\mathrm{L} / \mathrm{R}$ with your $\mathrm{L} / \mathrm{R}$ hand. R/L SCM: Support your head from behind on the 
L/R side with your L/R hand to prevent your neck flexors from having to work to keep your head up. Side bend your head to the L/R. Rotate your head slightly to the R/L. Extend your neck slightly until you feel a mild stretch, letting the weight of your head rest in your L/R hand $[28,29]$.

\section{Statistical Analysis}

The Kolmogorov-Smirnov test was used to confirm the conformity of digital measurements with the normal distributions. The SPSS statistical package (Version 11.0 for Windows) was used to analyze the obtained data. Mean and standard deviations were used in descriptive statistics. In the statistical analysis, the t-test and the $X^{2}$ test were used to determine the differences between sociodemographic characteristics. In the intra-group comparisons, the paired samples t-test was used. Also, the Mann Whitney U test was used in the inter-group analyses.

Table I. Socio-demographic data of the volunteers

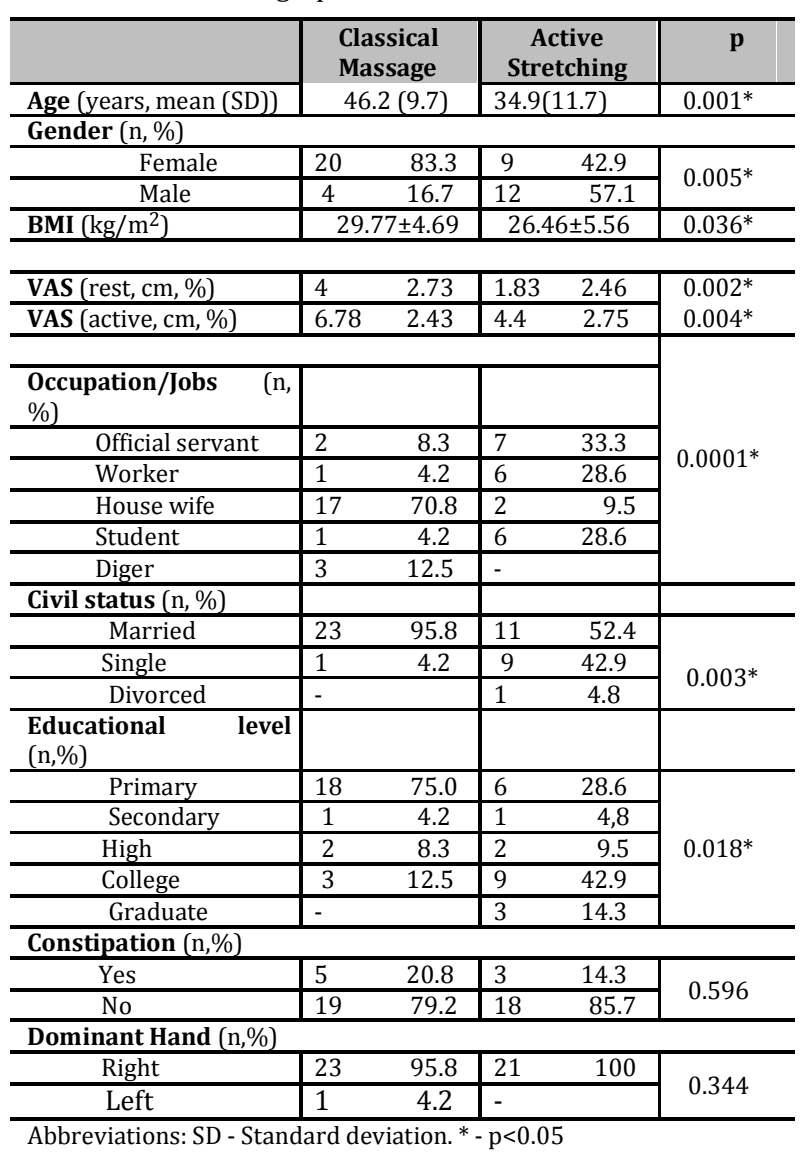

In the comparison of demographic data and PAAQ one-way analysis of variance was used. The relationship between VAS, PT average values and EMG-BF was detected with correlation analysis.

\section{Results}

Figure I shows the participant flow according to the CONSORT guidelines [30]. The classical massage group comprised 24 participants (53.3\%), while the active stretching group consisted of 21 participants (46.6\%).

Table I shows the socio-demographic information of the participants. In comparisons between the classical massage and stretching groups, statistically significant differences were found in terms of age, genders, BMI, VAS (relaxation/activity), occupation, marital status and educational status $(\mathrm{p}<0.05)$. No significant difference was found between these two groups in terms of dominant hand and constipation $(\mathrm{p}>0.05)$.

Table II. Comparison of Physical Activity

AssessmentQuestionnaire (PAAQ) values between two groups

\begin{tabular}{|c|c|c|c|c|c|}
\hline $\begin{array}{c}\text { Physical } \\
\text { activity } \\
\text { (MET/week) }\end{array}$ & n & $\begin{array}{c}\text { Classical } \\
\text { Massage } \\
\% \\
\end{array}$ & & $\begin{array}{l} \\
\% \\
\end{array}$ & $\mathbf{p}$ \\
\hline \multicolumn{5}{|l|}{ Work } & \multirow{4}{*}{$>0.05$} \\
\hline$<3$ & 2 & 66.7 & 3 & 23.1 & \\
\hline $3-6$ & & & 9 & 69.2 & \\
\hline$>6$ & 1 & 33.3 & 1 & 7.7 & \\
\hline \multicolumn{6}{|l|}{ School } \\
\hline$<3$ & 1 & 100 & 4 & 50.0 & \multirow[b]{3}{*}{$>0.05$} \\
\hline $3-6$ & - & & 2 & 25.0 & \\
\hline$>6$ & - & & 2 & 25.0 & \\
\hline \multicolumn{6}{|l|}{$\begin{array}{l}\text { Transportati } \\
\text { on }\end{array}$} \\
\hline$<3$ & 9 & 39.1 & 19 & 95.0 & \multirow{3}{*}{$0.000^{*}$} \\
\hline $3-6$ & 14 & 60.9 & 1 & 5.0 & \\
\hline$>6$ & - & & - & & \\
\hline \multicolumn{6}{|l|}{ House work } \\
\hline$<3$ & 23 & 95.8 & 19 & 100 & \multirow{3}{*}{$>0.05$} \\
\hline $3-6$ & 1 & 4.2 & - & & \\
\hline$>6$ & & & & & \\
\hline \multicolumn{6}{|c|}{ House work while sitting } \\
\hline$<3$ & 24 & 100 & 20 & 100 & \multirow{3}{*}{$>0.05$} \\
\hline $3-6$ & - & & - & & \\
\hline$>6$ & - & & - & & \\
\hline \multicolumn{6}{|c|}{ Leisure time activity } \\
\hline$<3$ & 14 & 93.3 & 6 & 85.7 & \multirow{3}{*}{$>0.05$} \\
\hline $3-6$ & 1 & 6.7 & 1 & 14.3 & \\
\hline$>6$ & - & & - & & \\
\hline \multicolumn{6}{|l|}{ Sports } \\
\hline$<3$ & - & & 2 & 20 & \multirow{3}{*}{$>0.05$} \\
\hline $3-6$ & 7 & 70 & 7 & 70 & \\
\hline$>6$ & 3 & 30 & 1 & 10 & \\
\hline \multicolumn{6}{|l|}{ Steps } \\
\hline$<3$ & 20 & 95.2 & 9 & 69.2 & \multirow{3}{*}{$0.05^{*}$} \\
\hline $3-6$ & 1 & 4.8 & 3 & 23.1 & \\
\hline$>6$ & - & & 1 & 7.7 & \\
\hline
\end{tabular}


The results of the physical activity assessment questionnaire are shown in Tables II and III. MET analysis of the energy consumed by the participants during a week shows a significant difference in the activities of transportation and climbing the stairs (respectively; $p=0.000$ and $\mathrm{p}=0.05$ ). There was a significant difference in the amount of energy (in kcal) consumed by the participants during a week between the groups in the activities of school, housework and climbing the stairs (respectively, $p=0.043, p=0.056$ and $\mathrm{p}=0.09$ ).

Only the EMG values on the right SCM muscles of the CM group were found to be significantly higher than the stretching group $(p=0.003)$. No significant difference was found between these two groups in terms of the PT average values of right and left SCM and TR muscles and EMG_BF average values of right and left TR and left SCM muscles ( $p>0.05$ ) (Table IV).

The correlation between VAS (activity/resting) and PAAQ was evaluated (Table V). In addition, we analyzed the relationship between VAS and EMG-BF averages, and PT average values. The pain was evaluated with VAS once more before the applications (Table V). Between the PT and BMI, only a significant positive relationship was found (Table V).

Comparing the gender and EMG_BF values, only a difference between SCM muscles and gender was found. SCM Right (R)/Left (L) EMG_BF values were found to be higher in women $(p=0,005$; $\mathrm{p}=0,000$ ) (Table VI). Comparing occupation and EMG_BF values, a difference between SCM L muscles and occupation was found $(p=0,003)$. The occupation with the highest mean was found to be housewife (Table VI).

In the comparison conducted between marital status and EMG-BF values it was observed that only SCM muscle was affected and the mean values of being married were found to have significantly higher EMG_BF SCM R/L $(p=0,50$ / 0,003) (Table VII).

Whether the physical activities in daily life are in correlation with pain and relaxation was evaluated in our study. In our study, the relationship between EMG-BF and PT average values and PAAQ was examined. It was detected that in most cases hobi met values were in correlation with PT values (Table VIII).

\begin{tabular}{|c|c|c|c|c|c|}
\hline \multirow[t]{2}{*}{ (kcal/week) } & \multicolumn{2}{|c|}{ Massage } & \multicolumn{2}{|c|}{ Stretching } & \\
\hline & n & $\%$ & n & $\%$ & \\
\hline \multicolumn{5}{|l|}{ Work } & \multirow{4}{*}{$>0.05$} \\
\hline$<1000$ & - & & - & & \\
\hline $1000-2499$ & - & & - & & \\
\hline$>2500$ & 3 & 100 & 13 & 100 & \\
\hline \multicolumn{6}{|l|}{ School } \\
\hline$<1000$ & - & & 1 & 12.5 & \multirow[b]{3}{*}{$0.043^{*}$} \\
\hline $1000-2499$ & 1 & 100 & - & & \\
\hline$>2500$ & - & & 7 & 87.5 & \\
\hline \multicolumn{6}{|l|}{ Transportation } \\
\hline$<1000$ & 18 & 78.3 & 17 & 85 & \multirow{4}{*}{$>0.05$} \\
\hline $1000-2499$ & 4 & 17.4 & 2 & 10 & \\
\hline$>2500$ & 1 & 4.3 & 1 & 5 & \\
\hline \multicolumn{5}{|l|}{ Sleeping } & \\
\hline$<1000$ & 1 & 4.2 & - & & \multirow{3}{*}{$>0.05$} \\
\hline $1000-2499$ & 3 & 12.5 & 2 & 9.5 & \\
\hline$>2500$ & 20 & 83.3 & 19 & 90.5 & \\
\hline \multicolumn{6}{|l|}{ House work } \\
\hline$<1000$ & 2 & 8.3 & 6 & 31.6 & \multirow{3}{*}{$0.05^{*}$} \\
\hline $1000-2499$ & 2 & 8.3 & - & & \\
\hline$>2500$ & 20 & 83.3 & 13 & 68.4 & \\
\hline \multicolumn{6}{|c|}{ House work while sitting } \\
\hline$<1000$ & 7 & 29.2 & 4 & 20 & \multirow{3}{*}{$>0.05$} \\
\hline $1000-2499$ & 8 & 33.3 & 5 & 25 & \\
\hline$>2500$ & 9 & 37.5 & 11 & 55 & \\
\hline \multicolumn{6}{|c|}{ Leisure time activity } \\
\hline$<1000$ & 8 & 53.3 & 2 & 28.6 & \multirow{3}{*}{$>0.05$} \\
\hline $1000-2499$ & 3 & 20 & 4 & 57.1 & \\
\hline$>2500$ & 4 & 26.7 & 1 & 14.3 & \\
\hline \multicolumn{6}{|l|}{ Sports } \\
\hline$<1000$ & 6 & 60 & 7 & 58,3 & \multirow{3}{*}{$>0.05$} \\
\hline $1000-2499$ & 2 & 20 & 3 & 25 & \\
\hline$>2500$ & 2 & 20 & 2 & 16.7 & \\
\hline \multicolumn{6}{|l|}{ Steps } \\
\hline$<1000$ & 24 & 100 & 13 & 100 & \multirow{3}{*}{$0,05^{*}$} \\
\hline $1000-2499$ & - & & - & & \\
\hline$>2500$ & - & & - & & \\
\hline
\end{tabular}


Table IV. Comparisons of Electromyography (EMG), Pain Threshold (PT) average values on the left and right ofSternocleidomastoideus (SCM) and Trapezius (TR) muscle between two groups

\begin{tabular}{|c|c|c|c|c|c|c|c|c|c|c|c|}
\hline & & \multicolumn{4}{|c|}{ Before application } & \multicolumn{5}{|c|}{ After application } & \multirow[b]{2}{*}{$\begin{array}{l}\text { VAS } \\
\text { Activity }\end{array}$} \\
\hline $\begin{array}{l}\text { Pain } \\
\text { Threshol } \\
\text { d (n=45) }\end{array}$ & & AGE & BMI & $\begin{array}{l}\text { VAS } \\
\text { Resting }\end{array}$ & $\begin{array}{l}\text { VAS } \\
\text { Activit } \\
\mathrm{y}\end{array}$ & $\begin{array}{l}\text { Pain } \\
\text { Threshold } \\
(n=45)\end{array}$ & & AGE & BMI & $\begin{array}{l}\text { VAS } \\
\text { Resting }\end{array}$ & \\
\hline TRR & $\begin{array}{l}\mathrm{r} \\
\mathrm{p}\end{array}$ & $\begin{array}{l}.169 \\
.267\end{array}$ & $\begin{array}{l}.388 \\
.009 *\end{array}$ & $\begin{array}{l}.254 \\
.093 \\
\end{array}$ & $\begin{array}{l}.067 \\
.660\end{array}$ & TR R & $\begin{array}{l}\mathrm{r} \\
\mathrm{p}\end{array}$ & $\begin{array}{l}.256 \\
.090\end{array}$ & $\begin{array}{l}.480 \\
.001 *\end{array}$ & $\begin{array}{l}.272 \\
.070\end{array}$ & $\begin{array}{l}.087 \\
.569 \\
\end{array}$ \\
\hline TR L & $\begin{array}{l}\mathrm{r} \\
\mathrm{p}\end{array}$ & $\begin{array}{l}.183 \\
.229 \\
\end{array}$ & $\begin{array}{l}.373 \\
.012 * \\
\end{array}$ & $\begin{array}{l}.262 \\
.082 \\
\end{array}$ & $\begin{array}{l}.115 \\
.453 \\
\end{array}$ & TR L & $\begin{array}{l}\mathrm{r} \\
\mathrm{p}\end{array}$ & $\begin{array}{l}.200 \\
.187 \\
\end{array}$ & $\begin{array}{l}.400 \\
.007 *\end{array}$ & $\begin{array}{l}.147 \\
.335 \\
\end{array}$ & $\begin{array}{l}.038 \\
.806 \\
\end{array}$ \\
\hline SCM R & $\begin{array}{l}\mathrm{r} \\
\mathrm{p}\end{array}$ & $\begin{array}{l}.131 \\
.391 \\
\end{array}$ & $\begin{array}{l}.237 \\
.116 \\
\end{array}$ & $\begin{array}{l}.158 \\
.300 \\
\end{array}$ & $\begin{array}{l}.099 \\
.519 \\
\end{array}$ & SCM R & $\begin{array}{l}\mathrm{r} \\
\mathrm{p}\end{array}$ & $\begin{array}{l}.008 \\
.958 \\
\end{array}$ & $\begin{array}{l}.295 \\
.050 * \\
\end{array}$ & $\begin{array}{l}.143 \\
.350 \\
\end{array}$ & $\begin{array}{l}.008 \\
.958 \\
\end{array}$ \\
\hline SCM L & $\begin{array}{l}\mathrm{r} \\
\mathrm{p}\end{array}$ & $\begin{array}{l}.088 \\
.566 \\
\end{array}$ & $\begin{array}{l}.219 \\
.148 \\
\end{array}$ & $\begin{array}{l}.138 \\
.366 \\
\end{array}$ & $\begin{array}{l}.117 \\
.444 \\
\end{array}$ & SCM L & $\begin{array}{l}\mathrm{r} \\
\mathrm{p}\end{array}$ & $\begin{array}{l}.044 \\
.774 \\
\end{array}$ & $\begin{array}{l}.295 \\
.050 * \\
\end{array}$ & $\begin{array}{l}.196 \\
.254 \\
\end{array}$ & $\begin{array}{l}.821 \\
.067 \\
\end{array}$ \\
\hline \multicolumn{12}{|c|}{ EMG_BF(n= 45) } \\
\hline TR R & $\begin{array}{l}\mathrm{r} \\
\mathrm{p}\end{array}$ & $\begin{array}{l}-.103 \\
.500\end{array}$ & $\begin{array}{l}-.022 \\
.884 \\
\end{array}$ & $\begin{array}{l}-. .035 \\
.821 \\
\end{array}$ & $\begin{array}{l}.081 \\
.597\end{array}$ & TRR & $\begin{array}{l}\mathrm{r} \\
\mathrm{p}\end{array}$ & $\begin{array}{l}.139 \\
.363 \\
\end{array}$ & $\begin{array}{l}-.203 \\
.181 \\
\end{array}$ & $\begin{array}{l}.132 \\
.388\end{array}$ & $\begin{array}{l}-.295 \\
.050 *\end{array}$ \\
\hline TR L & $\begin{array}{l}\mathrm{r} \\
\mathrm{p}\end{array}$ & $\begin{array}{l}.070 \\
.647\end{array}$ & $\begin{array}{l}. .147 \\
.334 \\
\end{array}$ & $\begin{array}{l}-.062 \\
.684 \\
\end{array}$ & $\begin{array}{l}.141 \\
.356 \\
\end{array}$ & TR L & $\begin{array}{l}\mathrm{r} \\
\mathrm{p}\end{array}$ & $\begin{array}{l}-.113 \\
.461 \\
\end{array}$ & $\begin{array}{l}.316 \\
.035 * \\
\end{array}$ & $\begin{array}{l}.021 \\
.891 \\
\end{array}$ & $\begin{array}{l}.213 \\
.159 \\
\end{array}$ \\
\hline SCM R & $\begin{array}{l}\mathrm{r} \\
\mathrm{p}\end{array}$ & $\begin{array}{l}.252 \\
.094 \\
\end{array}$ & $\begin{array}{l}.053 \\
.731 \\
\end{array}$ & $\begin{array}{l}.268 \\
.075 \\
\end{array}$ & $\begin{array}{l}.308 \\
.040 * \\
\end{array}$ & SCM R & $\begin{array}{l}\mathrm{r} \\
\mathrm{p}\end{array}$ & $\begin{array}{l}.175 \\
.250 \\
\end{array}$ & $\begin{array}{l}.086 \\
.576 \\
\end{array}$ & $\begin{array}{l}.151 \\
.323 \\
\end{array}$ & $\begin{array}{l}.204 \\
.179 \\
\end{array}$ \\
\hline SCM L & $\begin{array}{l}\mathrm{r} \\
\mathrm{p}\end{array}$ & $\begin{array}{l}.261 \\
.083\end{array}$ & $\begin{array}{l}.084 \\
.581\end{array}$ & $\begin{array}{l}.392 \\
.008 *\end{array}$ & $\begin{array}{l}.254 \\
.092\end{array}$ & SCM L & $\begin{array}{l}\mathrm{r} \\
\mathrm{p}\end{array}$ & $\begin{array}{l}.106 \\
.487\end{array}$ & $\begin{array}{l}. .173 \\
.255\end{array}$ & $\begin{array}{l}.007 \\
.966\end{array}$ & $\begin{array}{l}.089 \\
.559\end{array}$ \\
\hline
\end{tabular}

*- < <0.05, TR - Trapezius, SCM - Sternocloideumasteoideus, R/L - Right/Left, SD - Standard Deviation EMG_BF - Electromyography-Biofeedback

Table V. Compared Pain Threshold, Elektromyography Biofeedback average values and Age,

\begin{tabular}{|c|c|c|c|c|c|c|}
\hline & \multicolumn{2}{|c|}{ Before application } & \multirow[t]{2}{*}{$\mathrm{p}$} & \multicolumn{2}{|c|}{ After application } & \multirow[t]{2}{*}{ p } \\
\hline & $\begin{array}{c}\text { Classical } \\
\text { Massage } \\
(\mathrm{n}=24) \\
\text { Mean } \pm \mathrm{SD}\end{array}$ & $\begin{array}{l}\text { Stretching } \\
(n=20) \\
\text { Mean } \pm S D\end{array}$ & & $\begin{array}{c}\text { Classical } \\
\text { Massage } \\
(n=24) \\
\text { Mean } \pm S D\end{array}$ & $\begin{array}{l}\text { Stretching } \\
(n=20) \\
\text { Mean } \pm S D\end{array}$ & \\
\hline \multicolumn{7}{|c|}{ Algometre/ L } \\
\hline SCM & $14.60 \pm 4.99$ & $15.42 \pm 6.15$ & 0.629 & $15.97 \pm 4.91$ & $45.31 \pm 111.28$ & 0.203 \\
\hline Trapezius & $14.37 \pm 4.94$ & $14.44 \pm 6.51$ & 0.966 & $14.81 \pm 4.80$ & $18.43 \pm 19.67$ & 0.391 \\
\hline \multicolumn{7}{|l|}{ Emg/L } \\
\hline SCM & $9.04 \pm 4.26$ & $8.90 \pm 5.6$ & 0.926 & $9.16 \pm 3.15$ & $10.0 \pm 17.40$ & 0.819 \\
\hline Trapezius & $6.04 \pm 6.56$ & $88.85 \pm 375.8$ & 0.286 & $7.58 \pm 7.16$ & $7.90 \pm 18.03$ & 0.936 \\
\hline \multicolumn{7}{|c|}{ Algometre/R } \\
\hline SCM & $14.72 \pm 4.46$ & $15.60 \pm 6.48$ & 0.599 & $15.65 \pm 8.37$ & $9.38 \pm 3.32$ & 0.229 \\
\hline Trapezius & $15.60 \pm 4.78$ & $14.92 \pm 6.63$ & 0.695 & $14.39 \pm 4.71$ & $18.79 \pm 18.67$ & 0.294 \\
\hline \multicolumn{7}{|l|}{ Emg/R } \\
\hline SCM & $13.62 \pm 4.65$ & $11.76 \pm 5.02$ & 0.204 & $15.50 \pm 8.71$ & $9.11 \pm 3.17$ & $\mathbf{0 . 0 0 3}^{*}$ \\
\hline Trapezius & $8.75 \pm 6.46$ & $28.76 \pm 93.06$ & 0.299 & $8.12 \pm 4.14$ & $6.71 \pm 1.90$ & 0.159 \\
\hline
\end{tabular}

Missing data: The data contain extreme values were removed to reach the correct value.* $-\mathrm{p}<0.05$

Table VI. Pain Threshold and Electromyography Biofeedback after application according todemographics characteristics (gender and occupations)

\begin{tabular}{|c|c|c|c|c|c|c|c|c|}
\hline \multirow[b]{2}{*}{$\begin{array}{l}\text { Pain } \\
\text { threshol } \\
\text { d }\end{array}$} & \multicolumn{3}{|l|}{ Gender } & \multicolumn{4}{|c|}{ Occupations } & \multirow[b]{2}{*}{$\mathrm{P}$} \\
\hline & $\begin{array}{c}\text { Women } \\
n=29 \\
\text { Mean } \pm \text { SD }\end{array}$ & $\begin{array}{l}\text { Men } \\
n=16 \\
\text { Mean } \pm \text { SD }\end{array}$ & $\mathrm{P}$ & $\begin{array}{l}\text { Officer } \\
\mathrm{n}=9 \\
\text { Mean } \pm \text { SD }\end{array}$ & $\begin{array}{l}\text { Worker } \\
\mathrm{n}=7 \\
\text { Mean } \pm \mathrm{SD}\end{array}$ & $\begin{array}{l}\text { Housewife } \\
\mathrm{n}=19 \\
\text { Mean } \pm \text { SD }\end{array}$ & $\begin{array}{l}\text { Other } \\
\mathrm{n}=10 \\
\text { Mean } \pm \text { SD }\end{array}$ & \\
\hline TR R & $13.49 \pm 4.4$ & $16.16 \pm 6.9$ & .123 & $14.63 \pm 6.0$ & $16.38 \pm 7.6$ & $13.63 \pm 3.7$ & $14.46 \pm 6.7$ & .748 \\
\hline TR L & $13.70 \pm 4.7$ & $15.95 \pm 7.0$ & .209 & $15.02 \pm 6.1$ & $16.18 \pm 7.7$ & $13.82 \pm 4.0$ & $14.14 \pm 7.0$ & .816 \\
\hline SCM R & $15.28 \pm 3.9$ & $17.07 \pm 6.3$ & .251 & $15.56 \pm 5.4$ & $18.25 \pm 5.6$ & $15.64 \pm 3.7$ & $15.13 \pm 6.2$ & .606 \\
\hline SCM L & $16.47 \pm 4.3$ & $16.68 \pm 7.0$ & .901 & $15.68 \pm 6.1$ & $20.58 \pm 5.7$ & $16.09 \pm 3.9$ & $15.37 \pm 6.2$ & .188 \\
\hline \multicolumn{9}{|l|}{ EMG_BF } \\
\hline TR R & $7.44 \pm 2.81$ & $7.50 \pm 4.22$ & .961 & $8.33 \pm 3.1$ & $6.42 \pm 1.1$ & $7.00 \pm 2.5$ & $8.30 \pm 5.3$ & .530 \\
\hline TR L & $6.37 \pm 5.5$ & $5.18 \pm 5.9$ & .507 & $4.66 \pm 2.1$ & $4.14 \pm 2.6$ & $5.36 \pm 3.5$ & $9.50 \pm 10.2$ & .151 \\
\hline SCM R & $14.79 \pm 7.7$ & $8.75 \pm 3.6$ & $.005^{*}$ & $9.33 \pm 2.0$ & $11.57 \pm 3.6$ & $14.68 \pm 3.9$ & $12.50 \pm 13.6$ & .311 \\
\hline SCM L & $9.17 \pm 3.0$ & $5.25 \pm 1.9$ & $.000 *$ & $5.88 \pm 1.3$ & $7.85 \pm 3.7$ & $9.68 \pm 3.0$ & $5.80 \pm 2.7$ & $.003^{*}$ \\
\hline
\end{tabular}


Timișoara Physical Education and Rehabilitation Journal

Table VII. Pain Threshold and Electromyography Biofeedback after application

\begin{tabular}{|c|c|c|c|c|c|c|}
\hline \multirow[b]{2}{*}{$\begin{array}{l}\text { Pain } \\
\text { Threshol } \\
\text { d }\end{array}$} & \multicolumn{2}{|c|}{ Marital status } & \multirow{2}{*}{$\mathrm{p}$} & \multicolumn{3}{|c|}{ Constipation } \\
\hline & $\begin{array}{c}\text { Married } \\
(\mathrm{n}=34) \\
\text { Mean } \pm \mathrm{SD}\end{array}$ & $\begin{array}{l}\text { Single } \\
(\mathrm{n}=11) \\
\text { Mean } \pm \text { SD }\end{array}$ & & \begin{tabular}{|l} 
Yes \\
$(\mathrm{n}=8)$ \\
Mean \pm SD
\end{tabular} & \begin{tabular}{|l} 
No \\
$(\mathrm{n}=37)$ \\
Mean $\pm S D$
\end{tabular} & $\overline{\mathrm{p}}$ \\
\hline TRR & $14.47 \pm 5.0$ & $14.35 \pm 7.0$ & .950 & $14.32 \pm 4.0$ & \begin{tabular}{|l|}
$14.47 \pm 5.8$ \\
\end{tabular} & .946 \\
\hline TR L & \begin{tabular}{|l|}
$14.54 \pm 5.2$ \\
\end{tabular} & $14.36 \pm 7.2$ & .928 & \begin{tabular}{|l|}
$13.78 \pm 4.6$ \\
\end{tabular} & $14.65 \pm 5.9$ & .701 \\
\hline SCM R & $15.91 \pm 4.3$ & $15.93 \pm 6.5$ & .719 & $15.62 \pm 2.2$ & $15.98 \pm 5.38$ & .854 \\
\hline SCM L & \begin{tabular}{|l|}
$16.71 \pm 5.0$ \\
\end{tabular} & $16.03 \pm 6.5$ & .970 & $15.13 \pm 4.0$ & $16.85 \pm 5.6$ & .418 \\
\hline \multicolumn{7}{|l|}{ EMG_BF } \\
\hline TRR & \begin{tabular}{|l|}
$7.41 \pm 2.82$ \\
\end{tabular} & $7.63 \pm 4.73$ & .849 & $7.25 \pm 3.1$ & $7.51 \pm 3.4$ & .842 \\
\hline TRL & $5.91 \pm 5.2$ & $6.09 \pm 7.0$ & .929 & $4.25 \pm 2.4$ & $6.32 \pm 6.1$ & .355 \\
\hline SCM R & \begin{tabular}{|l|}
$13.82 \pm 7.6$ \\
\end{tabular} & $9.00 \pm 3.6$ & $.050^{*}$ & $12.25 \pm 5.0$ & $12.72 \pm 7.5$ & .866 \\
\hline SCM L & \begin{tabular}{|l|}
$8.58 \pm 3.3$ \\
\end{tabular} & $5.27 \pm 1.6$ & $.003^{*}$ & \begin{tabular}{|l|}
$7.12 \pm 2.5$ \\
\end{tabular} & \begin{tabular}{|l|}
$7.91 \pm 3.4$ \\
\end{tabular} & .544 \\
\hline
\end{tabular}

SD - Standard Deviation EMG_BF - Electromyography-Biofeedback

\section{Discussion}

This is the first study examining the acute effect of classical massage and stretching exercises on pain and relaxation. In addition to this study suggest that physical activities (specially steps, sitting home working and school activities) effects on neck pain and SCM muscle, especially.

As a result of this study, we found that in a comparison of the effectiveness of one-session application of two physiotherapy methods, selfstretching and CM, showed that self-stretching is more effective than $\mathrm{CM}$ in providing muscle relaxation. The role of conservative treatments for managing neck pain is not clear. Health professionals should attempt to identify possible cognitive, behavioral, demographic, organizational or practical barriers which may impact on patient adherence to the treatment [31]. In the study, we wanted to compare the two applications we conducted while investigating the effect of physical activity on the muscles and wanted to measure EMG-BF and PT.

EMG-BF is influenced by a number of factors including: Series elastic components, ability of the action potential of propagate, and excitationcontraction coupling. Elongation of decrease in EMG BF values is believed to be due to mechanical stress placed on the muscle and increased passive tension on non-contractile structures [32,33]. Behm DG et al.[32] (2013) studied how to evaluate changes in neural and evoked muscle responses with massage and static stretching. They found that static stretching increased the duration of electromechanical delay whereas massage had no effect on this property. But the static stretching induced Hreflex depression during and 30s following massage and recovered rapidly once the stretching ceased. Electromechanical delay was generally prolonged when massage was combined with stretching [32]. McBridge et al. [34] (2007) investigated EMG activity during the proprioceptive neuromuscular facilitation stretching method and observed EMG activity. However, their results should be considered with caution, since they proposed that passive stretching may be safer than active techniques. Indeed, $88 \%$ of their volunteers reported that passive stretching procedures were more comfortable [35].

According to Soysal M et al. [7] (2012), there is a correlation between physical activity and neck pain. Sleeping status of the individual, whether in depression or not, affects the life quality. They found that quality of life scores of outpatients were significantly negatively correlated with physical activity. Recent studies show that patients with spinal pain have impaired daily activities $[7,36,37,38]$. There are many studies $[7,39,40,41]$ in the literature about neck pain but few studies that compare physical activity and its effectiveness on muscles of areas of the neck in patients with neck pain.

Among persons with neck pain little is known from previous studies about the potential positive effects of physical activity on the prognosis of neck pain, and high-quality studies are scarce. ${ }^{41}$ A Dutch study summarized that active time was associated favorably with neck and shoulder symptoms and with sickness in a working cohort [42]. This was also found in two other studies $[43,44]$ reporting positive associations between physical activity level 
and neck pain. Hildebrandt et al. [44] (2000) suggested that in a sedentary working situation, higher physical activity level seems to be positive for spinal pain. According to Shan $\mathrm{Z}$ et al. [40] (2013) in their study there is no association between physical activity and neck/shoulder pain.

In our study, in parallel with the literature, some demographic characteristics (being women, being housewife, being married, BMI) had an effect on neck pain. No relationship was found between age and PT or EMG-BF values. According to Croft PR et al [45]. (2001) there is no apparent link between age and weight and neck pain but there is relative to a past history of neck pain. Rasmussen Barr E et al. [41] (2013) found that between levels of physical activity and neck pain in active women compared to those with sedentary leisure time, indicated a positive prognostic effect, whereas BMI was not a prognostic factor. No such associations were found in men. Shan Z et al. [40] (2013) suggest that prevalence of neck pain was significantly higher in girls compared to boys, which is consistent with adults.

Pedersen MM et al.[46] (2013) stated that problems experienced frequently in the workplace involved neck pains and that with slight and effective exercises in the workplace neck pain complaints decreased. In this study, we did not evaluate the self-efficiency (the adaptation to exercise) of the individuals. However, in our study with stretching performed for a unique session the decrease in EMG-BF values on the SCM muscle cannot be ignored. The most important problems for exercising in the workplace are time, other employees and venue [46]. For this reason, we think that muscle relaxation obtained through a unique session will increase adaptation to the exercise.

Veiersted KB et al [47] (1993) used EMG-BF values on TR muscle and stated that in workplace studies, one of the muscles creating neck pain is the trapeze muscle. In our study, no correlation was found between workplace activities and trapeze muscle EMG-BF and PT values. In conclusion, in all these analyses, in almost all activities, it was observed that mostly the SCM muscle was influenced.

In this study, we showed that the stretching group consumed more energy during school and work activities (desk work activities) and the classical massage group consumed more energy during housework and spare time activities (activities including mass body movements) [48], while the levels of energy consumed during sporting activities were similar in both groups. As indicated in previous studies, neck pain complaints are more common in desk work activities [49]. As the stretching group took place in desk work activities in our study, neck and upper back muscles were used more extensively compared to the CM group. This may be the reason behind the higher success of the treatment in the stretching group.

According to the results we obtained, it is interesting that there are both positive and negative relationships between physical activity and after application PT and EMG-BF values.

Our prediction for negative correlation is as follows: Generally after stretching and massage, a decrease in EMG-BF values is expected indicating muscle relaxation. Increased flexibility caused by the viscoelastic properties of muscles may have led to a reduced muscle spindle response, with a consequent reduction of the EMG-BF signal [50]. Massage may produce an increase in peripheral circulation, resulting in general relaxation and relief. $^{32,50}$ Especially after the application, the positive correlation between the EMG-BF values and physical activity found reveals the increase in EMG-BF values of the applications performed. A hypothesis explaining this could be based on changes in the relationship between length and tension. If stretching really induces myofibrillogenesis, plastic changes in muscle structure would alter the length-tension relationship. Improved interaction between actomyosin bridges may have led to better muscle recruitment, increasing EMG-BF activity. As massage techniques are transmitted to the muscle through the skin, the activity of the cutaneous afferents would be expected to play a role in the excitation of the central nervous system [51]. Hence muscle relaxation can be quite variable dependent upon the type, duration and location of the stimulation. ${ }^{32}$

With all of these factors, our hypothesis for negative and positive relationships seen can be related to the posture in the activities performed by the individual. In our study, especially without looking at the evaluations between PT and free time activities (hobby), a positive correlation was found. 
Tettamanti A et al. [52] (2013) found that the characteristics of postural activity derive from the mechanical characteristics of the body masses that are moved. For example, during abduction of the upper limb, the scapula has to be oriented upward and must remain in contact with chest, whereas the agonist muscles tend to produce a movement in a downward direction. The muscles in postural stabilization prevent unwanted movement of the cervical-dorsal spine. The trunk is stabilized by contra-lateral movement and rotational torque is avoided. Along each ROM, if the action is done wrongly by the upper extremity, it may cause injuries with the effects of ground reactions. It may cause pain [52]. In addition, in the activities made again in the posture, it may cause pain by affecting muscle balance $[53,54]$. In this study, the postures of the individuals were not evaluated. This situation may be seen as a limitation of our study. In future studies, the correlation between PAAQ and posture should be explored.

Besides this, we estimated that the correlation between hobby and pain is due to the fact that while the individuals are pursuing enjoyable activities they like, they do not pay attention to their posture and spend a lot of time in the wrong position (spending a long time standing for fishing, watching TV, knitting etc.). In our study, in accordance with the information obtained from the participants, most of the free time activities are sedentary. In the literature, it is stated that sedentary activity in leisure time is associated with higher prevalence rates of low back symptoms and sick leave due to low back symptoms [44].

There are some limitations to this study. The investigation of whether the participants had taken analgesia and muscle relaxants before the applications could affect our results. In our study, we did not ask whether the individuals had neck pain or not. Cross sectional studies report that recalled injury is more common in those with neck pain [45]. The type or nature of injury may be important. Road traffic related whiplash injury cannot be assumed to be equivalent to occupational trauma (overuse); sports injury and domestic accidents are other possible causes. An important limitation of this study was the small sample size.

It is known that the self-report method used in physical activity evaluations and in BMI calculations may result in faults $[41,42]$. Our study is important in terms of investigating the correlation between physical activity and musculoskeletal disorders via physical evaluation survey and has validity and reliability. In the literature only certain activities (the activity conducted by sitting in the workplace $^{55}$, during computer activities ${ }^{40}$ were focused on the correlation between physical activity and pain. In our study, only the EMG-BF value of either the SCM muscle [39] or TR muscle [47] were used. In our study (as suggested by Gorman E et al. [55] (2013)), all physical activities were mentioned and both SCM and TR muscles were included. Findings are discussed in light of the sense of coherence, physical activity, and positive and negative effects, as in the literature. Along with long term monitoring of neck pain in the future, we suggest the evaluation of EMG-BF values of the neck muscles.

\section{Conclusions}

Comparison of the effectiveness of one-session application of two physiotherapy methods, selfstretching and CM, showed that self-stretching is more effective than $\mathrm{CM}$ in providing upper back muscle relaxation. One-session application of selfstretching showed its effectiveness on SCM muscle relaxation. As individuals can easily apply selfstretching within daily routines in the work place, this may reduce clinical visits, thus helping to reduce health expenses. Physical activities (especially steps, sitting at home working and school activities) particularly affect neck pain and SCM muscle. Evaluation of parameters affecting the level of physical activity is very important and should be considered in planning the therapy programs of patients so that they can return to their normal daily lives.

\section{References}

1. Viljanen M., Malmivaara A., Uitti A., Rinne M., Palmroos P., Laippala P. (2003) Effectiveness of dynamic muscle training,relaxation training, or ordinary activity for chronic neck pain: randomised controlled trial. BMJ, 327 (7413):475. 2. Kim H., Geiger-Brown, Trinkoff J., Muntaner A.C. (2010) Physically demanding workloads and the risks of musculoskeletal disorders in homecare workers in the USA. Health Soc Care Community, 18(5):445-45.

3. Goode A.P., Freburger J., Carey T. (2010) Prevalence, practicepatterns and evidence for chronic neck pain. Arthritis Care Res,62(11):1594-1601. 
4. Mork P.J., Westgaard R.J. (2009) Back posture and low back muscle activity in female computer workers: A field study. ClinBiomech, 24:169-175.

5. Guez M., Hildingsson C., Nilsson M., Toolanen G. (2002) The prevalence of neck pain: a population-based study from northern Sweden. Acta Orthop Scand, 73(4):455-9.

6. Marquez D.X., Hoyem R., Fogg L., Bustamente E.E., Staffileno B., Wilbur J.E. (2011) Physical activity of urban community-dwelling older laino adults. J Phys Act Health, 8(2):161-107.

7. Soysal M., Kara B., Arda N. (2013) Assessment of PhysicalActivity in Patients with Chronic Low Back on Neck Pain. Turk Neurosurg, 23(1):75-80.

8. Geisser M.E., Robinson M.E., Miller Q.L., Bade S.M. (2003) Psychosocial factors and functional capacity evaluation amng persons with chronic pain. J Occup Rehabil, 13:259-276.

9. Özcan E., Öztürk Y., Dinçer N., Sezen K., Berker E. (2003) Kronik Boyun Ağrısında Akupunkturun Etkinliği Ön Çalışma. Turk. Fiz. Tip Rehab, 49(6).

10. Zusman M. (2012) A note to the musculoskeletalphysiotherapist. J Back Musculoskelet Rehabil, 25(2):103-7.

11. Moffett J., McLean S. (2006) The role of physiotherapy in themanagement of non-specific back pain and neck pain. Review.Rheumatology, 45:371-378.

12. Schonstein E., Kenny D., Keating J., Koes B., Herbert R.D. (2003) Physical conditioning programs for workers with back andneck pain: a Cochrane systemic review. Spine, 28:E391-E395. 13. Ritvanen T., Zaproudina N., Nissen M., Leinonen V., Hanninen O. (2007) Dynamic Surface Electromyographic Responses In Chronıc Low Back Pain Treated By Traditional Bone Setting And Conventional Physical Therapy. J Manipulative Physiol Ther, 30: 31-37. 14. Lariviere C., Arsenault A.B., Gravel D., Gagnon D., Loisel P. (2002) Evaluation of measurement strategies to increase thereliability of EMG indices to assess back muscle fatigue and recovery. J Electromyogr Kinesiol, 12: 91-102.

15. Holtermann A., Søgaard K., Christensen H., Dahl B., Blangsted A.K. (2008) The influence of biofeedback training on trapeziusactivity and rest during occupational computer work: a randomized controlled. Eur J Appl Physio, 104:983-989.

16. Brattberg G. (1999) Connective tissue massage in thetreatment of fibromyalgia. Eur J Pain 1999; 3: 235-245

17. Bretischwerdt C, Cano R.L., Cerro L.P., Penas C.F., Sendin F.A. (2010) Immediate effects of hamstring muscle stretching onpressure pain sensitivity and acti.ve mouth opening in healthy subjects. J Manipulative Physiol Ther, 33:42-47.

18. Furlan A.D., Yazdi F., Tsertsvadze A., Gross A., Van Tulder M. Santaguida L., Gagnier J., Ammendolia C., Dryden T., Doucette S., Skidmore B., Daniel R., Ostermann T., Tsouros S. (2012) Asystematic review and meta-analysis of efficacy, costeffectiveness, and safety of selected complementary and alternative medicine for neck and low-back pain. Evid Based Complement Alternat Med,953139.

19. Haraldsson B.G., Gross A.R., Myers C.D., Ezzo J.M., Morien A., Goldsmith C., Peloso P.M., Bronfort G. (2006) Cervical OverviewGroup. Massage for mechanical neck disorders. Cochrane DatabaseSyst Rev, 19(3):1-75.

20. Raijmakers B.G., Nieuwenhuizen M.G., Beckerman H., de Groot S. (2014) Differences in the course of daily activity levelbetween persons with and without chronic pain.Am J Phys MedRehabil. 00:00-00.

21. Ohrbach R., Gale E.N. (1989)Pressure pain thresholds, clinicalassessment, and differential diagnosis: reliability and validity in patients with myogenic pain. Pain, 39: 157-169.

22. Okifuji A., Türk D., Sinclair J., Starz T., Marcus D. (1997) Astandardized manual tender point survey. I. Development and determination of a threshold point for the identification of positive tender points in fibromyalgia syndrome. J Rheumatol, 24: 377-383.

23. Soderberg G.L., Cook T.M. (1984) Electromyography inbiomechanics. Electromyography in biomechanics. Phys Ther, 64:12.

24. Ross R.G., Lastayo P.C. (1997) Clinic Assessment of Pain. InDeusen J.V., Brunt D. - Assessment in occupational therapy and physical therapy. Philadelphia: W.B. Saunders Company, 123133.

25. Karaca A., Ergen E., Koruc Z. (2000) Fiziksel aktivitedeğerlendirme anketi (FADA) güvenirlik ve geçerlik çalışması. SBD11: 17-28.

26. Ainsworth B.E., Haskell W.L., Whitt M.C., Irwin M.L., Swartz A.M., Strath S.J. (2000) Compendium of physical activities: anupdate of activity codes and MET intensities. Med. Sci. SportsExerc 32:9:498-516.

27. Geoffrey C. Goats. (1994) Review. Massage - the scientificbasis of an ancient art: part 2. Physiological and therapeutic effects. Br J Sp Med, 28(3).

28. Özer D., Baltacı G. (2008) İş Yerinde Fiziksel Aktivite Kitapçığı, Hacettepe Üniversitesi Sağlı Bilimleri Fakültesi Fizik Tedavi ve Rehabilitasyon Bölümü, Klasmat Matbaacılık, ISBN: 978-975-590246-3

29. Hanten W.P., Olson S.L., Butts N.L., Nowicki A.L. (2000) Effectiveness of a Home Program of Ischemic Pressure Followed by Sustained Stretch for Treatment of Myofascial Trigger Points. PhysTher, 80(10):997-1003.

30. Begg C., Cho M., Eastwood S., et al. (1996) Improving the quality of reporting of randomized controlled trials. The CONSORTstatement. JAMA, 276:637-9.

31. McLean S.M., Moffett K. J.A., Sharp D.M., Gardiner E. (2013) Arandomised controlled trial comparing graded exercise treatment and usual physiotherapy for patients with non-specific neck pain (the GET UP neck pain trial). Man Ther, 18:199-205. 32. Behm D.G., Peach A., Maddigan M., Aboodarda S.J., DiSanto M.C., Buton D.C., Maffiuletti N.A. (2013) Masssage and stretchingreduce spinal reflex excitability without affecting twitch contractile properties. J Electromyogr Kinesiol. 23:12151221.

33. Howatson G. (2010) The impact of damaging exercise on electromechanical delay in biceps brachii. J ElectromyogrKinesiol. 20(3):477-81.

34. McBride J.M., Deane R. and Nimphius S. (2007) Effect ofstretching on agonist-antagonist muscle activity and muscle force output during single and multiple joint isometric contractions. Scand J Med Sci Sports, 17(1):54-60.

35. Marques A.P., Vasconcelos A.A.P., Cabral C.M.N., Sacco I.C.N. (2009). Effect of frequency of static stretching on flexibility,hamstring tightness and electromyographic acivity. Braz J MedBiol Res, 42(10):949-953.

36. Koes B. ( 2010) Moderate quality evidence that compared toadvice to rest in bed, advice to remain active provides small improvements in pain and functional status in people with acute low back pain. Evid Based Med, 15(6):171-2.

37. Bousema E.J., Verbunt J.A., Seelen H.A., Vlaeyen J.W., Knottnerus J.A. (2007) Disuse and physical deconditioning inthe first year after the onset of back pain. Pain, 130(3):279-86. 38. Van Weering M., Vollenbroek-Hutten M.M., Kotte E.M., Hermens H.J. (2007) Daily physical activities of patients withchronic pain or fatigue versus asymptomatic controls. A systematic review. Clin Rehabil, 21(11):1007-23.

39. Bakar Y., Sertel M., Öztürk A., Yümin E.T., Tatarlı N., Ankaral H. (2014) Short term effects of classic massage compared toconnective tissue massage on pressure pain threshold and muscle relaxation response in women with chronic neck pain: A preliminary study. J Manipulative and Physiol Ther, 37:415-421.

40. Shan Z., Deng G., Li J., Li Y., Zhang Y., Zhao Q. (2013) Correlation analysis of neck/shoulder pain and low back pain with the use of digital products, physical activity and psychological status among adolescents in shanghai. Plos one, 8(10):e78109.

41. Rasmussen-Barr E., Bohman T., Hallqvist J., Holm L.W., Skillgate E. (2013) Do physical activity level and body mass indexpredict recovery from persistent neck pain in men and women of working age? A population-based cohort study. Eur Spine J,22:2077-2083.

42. Van den Heuvel S.G., Boshuizen H.C., Hildebrandt V.H., Blatter B.M., Ariëns G.A., Bongers P.M. (2005) Effect of sporting activityon absenteeism in a working population. Br J Sports Med, Mar;39(3): e15.

43. Miranda H., Viikari-Juntura E., Martikainen R., Takala E.P. Riihimäki H. (2001) Physical exercise and musculoskeletal pain among forest industry workers. Scand J Med Sci Sports, 11(4):239-46

44. Hildebrandt V.H., Bongers P.M., Dul J., van Dijk F.J., Kemper H.C. (2000) The relationship between leisure time, physicalactivities and musculoskeletal symptoms and disability in worker populations. Int Arch Occup Environ Health, Nov;73(8):507-18.

45. Croft P.R., Lewis M., Papageorgiou A.C., Thomas E., Jayson M.I.V., Macfarlane G., Silman A.J. (2001) Risk factors for neck pain:a longitudinal study in the general population. Pain, 93:317325.

46. Pedersen M.M., Zebis M.K., Langberg H., Poulsen O.M., 
Mortensen O.S., Jensen J.N., Sjogaard G., Bredahl T., Andersen L.L. (2013) Influence of self-efficacy on compliance toworkplace exercise. Int J Behav Med, Sep;20(3):365-70.

47. Scand J Work Environ Health. 1993 Aug;19(4):284-90. (1993) Electromyographic evaluation of muscular work pattern asa predictor of trapezius myalgia. Scand J Work Environ Health,19(4):284-90.

48. Kallenberg L.A.C., Hermens H.J., Vollenbroek-Hutten M.M.R. (2006) Distinction between computer workers with and withoutwork-related neck shoulder complaints based on multiple surface EMG parameters. Int J Ind Ergon, 36:921-929.

49. Pettersen V., Westgaard R.H. (2004) The association betweenupper trapezius activity and throax movement in classical singing. J Voice, 18(4):500-512.

50. Kassolik K., Jaskolska A., Kisiel-Sajewicz K., Marusaki J., Kawczynski A., Jaskolski A. (2009) Tensegrity principle inmassage demonstrated by electro and mechanomyography. JBodyw Mov Ther, 13:164-170.

51. Sayenko D.G., Vette A.H., Obata H., Alekhina M.I., Akai M.,
Nakazawa K. (2009) Differential effects of plantar cutaneousafferent excitation on soleus stretch and H-reflex. MuscleNerve. Jun;39(6):761-9.

52. Tettamanti A., Giordano M., Gatti R. (2013) Effects of coupledupper limbs movements on postural stabilisation. J ElectromyogrKinesiol, 23:1222-1228.

53. Franz M., Zenk R., Vink P., Hallbeck S. (2011) The effect of alightweight massage system in a car seat on comfort and electromyogram. J Manipulative Physiol Ther, 34:107-113.

54. Lima B.N., Lucareli P.R.G., Gomes W.A., Silva J.J., Bley A.S. Hartigan E.H., Marchetti P.H. (2014) The acute effects of unilateralankle plantar flexors static-stretching on postural sway and gastrocnemius muscle activity during single-leg balance tasks. J Sports Sci Med, 13:564-570.

55. Gorman E., Ashe M.C., Dusntan D.W., Hanson H.M., Madden K., Wİnkler E.A.H., McKay H.A., Healy G.N. (2013) Does an activitypermissive workplace change office workers sitting and activity time? Plos On, 8(10): e76723. 